\title{
Conclusions.
}

The site has proved to be the most adequate of the Argentinian Antarctic stations. There are some periods of very good observing conditions, as long as 126 hours, but it is difficult to develop a good observing program due to the uncertainty when these periods occur. During the good periods the turbulence and transparency of the sky are adequate for astronomical work, but auroral phenomena are very frequent. We think that the best place for astronomical observations should be far from the coast and the auroral zone. We will continue the work with a less subjective method than the one presented and with appropriate instruments.

Is Antarctica attractive for astronomical work? If there is some place with a stable climate, at high altitude, accessible at least each 2 months, with small snow accumulation and with a laminar regime of winds, we say YES! We think it is important to establish an astronomical observatory in the Antarctic.

\section{DAYTIME ASTRONOMICAL OBSERVING CONDITIONS AT SOUTH POLE}

\author{
J Harvey \\ National Solar Observatory, Tucson
}

The Sun has been observed at South Pole nearly every austral summer since 1979. This experience shows that the duration of uninterrupted observations is limited by clouds to runs of at best $\sim 150$ hours. Impressively high duty cycles can be achieved over longer periods. Sky clarity is often superb but even in cloudless conditions, ice crystal precipitation storms can be a problem. Daytime observations of the Sun and bright stars show that visible seeing quality is limited to about 2-3 arc seconds at altitudes of 15-20 degrees. Nearer the zenith, the seeing quality approaches 1 arc second in light wind. Seeing quality appears to vary with wind speed and direction and also diurnally with changing solar illumination of the surface ridges (sastrugi). Seeing is degraded by turbulence in the exceptionally large temperature gradient in the first few hundred meters above the surface. There are suggestions that both daytime and, perhaps more so, nighttime cloudiness increased over the last decade. This may be related to increasing amounts of $\mathrm{CO}_{2}, \mathrm{CH}_{4}$ and CFCs in the polar atmosphere. 